\title{
Hybrid quantum circuit with a superconducting qubit coupled to a spin ensemble
}

\author{
Y. Kubo ${ }^{1}$, C. Grezes ${ }^{1}$, A. Dewes ${ }^{1}$, T. Umeda ${ }^{2}$, J. Isoya ${ }^{2}$, H. Sumiya ${ }^{3}$, N. \\ Morishita $^{4}$, H. Abe ${ }^{4}$, S. Onoda ${ }^{4}$, T. Ohshima ${ }^{4}$, V. Jacques ${ }^{5}$, A. Dréau ${ }^{5}$, \\ J.-F. Roch ${ }^{5}$, I. Diniz ${ }^{6}$, A. Auffeves ${ }^{6}$, D. Vion ${ }^{1}$, D. Esteve ${ }^{1}$, and P. Bertet ${ }^{1}$ \\ ${ }^{1}$ Quantronics group, SPEC (CNRS URA 2464), IRAMIS, \\ DSM, CEA-Saclay, 91191 Gif-sur-Yvette, France \\ 2 Research Center for Knowledge Communities, \\ University of Tsukuba, Tsukuba 305-8550, Japan \\ ${ }^{3}$ Sumitomo Electric Industries Ltd., Itami 664-001, Japan \\ 4 Japan Atomic Energy Agency, Takasaki 370-1292, Japan \\ ${ }^{5}$ LPQM (CNRS UMR 8537), ENS de Cachan, 94235 Cachan, France and \\ 6 Institut Néel, CNRS, BP 166, 38042 Grenoble, France
}

(Dated: October 29, 2018) 
Present-day implementations of quantum information processing rely on two widely different types of quantum bits (qubits). On the one hand, microscopic systems such as atoms or spins are naturally well decoupled from their environment and as such can reach extremely long coherence times [1, 2]; on the other hand, more macroscopic objects such as superconducting circuits are strongly coupled to electromagnetic fields, making them easy to entangle [3, 4] although with shorter coherence times [5, 6]. It thus seems appealing to combine the two types of systems in hybrid structures that could possibly take the best of both worlds. Here we report the first experimental realization of a hybrid quantum circuit in which a superconducting qubit of the transmon type [5, 7] is coherently coupled to a spin ensemble consisting of nitrogen-vacancy (NV) centers in a diamond crystal [8] via a frequency-tunable superconducting resonator [9] acting as a quantum bus. Using this circuit, we prepare arbitrary superpositions of the qubit states that we store into collective excitations of the spin ensemble and retrieve back later on into the qubit. We demonstrate that this process preserves quantum coherence by performing quantum state tomography of the qubit. These results constitute a first proof of concept of spin-ensemble based quantum memory for superconducting qubits [10-12]. As a landmark of the successful marriage between a superconducting qubit and electronic spins, we detect with the qubit the hyperfine structure of the NV center.

Superconducting qubits have been successfully coupled to electromagnetic [13] as well as mechanical [14 resonators; but coupling them to microscopic systems in a controlled way has up to now remained an elusive perspective - even though qubits sometimes turn out to be coupled to unknown and uncontrolled microscopic degrees of freedom with relatively short coherence times [15]. Whereas the coupling constant $g$ of one individual microscopic system to a superconducting circuit is usually too weak for quantum information applications, ensembles of $N$ such systems are coupled with a constant $g \sqrt{N}$ enhanced by collective effects. This makes possible to reach a regime of strong coupling between one collective variable of the ensemble and the circuit. This collective variable, which behaves in the low excitation limit as a harmonic oscillator, has been proposed [10 12] as a quantum memory for storing the state of superconducting qubits. Experimentally, the strong coupling between an ensemble of electronic spins and a superconducting resonator has been demonstrated 
spectroscopically [16 18], and the storage of a microwave field into collective excitations of a spin ensemble has been observed very recently [19, 20]. These experiments were however carried out in a classical regime since the resonator and spin ensemble behaved as two coupled harmonic oscillators driven by large microwave fields. In the perspective of building a quantum memory, it is instead necessary to perform experiments at the level of a single quantum of excitation. For that purpose, we integrate for the first time on the same chip three different quantum systems : an ensemble of electronic spins, a superconducting qubit, and a resonator acting as a quantum bus between the qubit and the spins. A sketch of the experiment is shown in Fig. 1 .

The spin ensemble $N V$ consists of $\sim 10^{12}$ negatively-charged NV color centers [8] in a diamond crystal. These centers have an electronic spin $S=1$, with electron spin resonance (ESR) transition frequencies $\omega_{ \pm}$between energy levels $m_{S}=0$ and $m_{S}= \pm 1$ of about $2.88 \mathrm{GHz}$ in zero magnetic field (see Fig. 1 c). The electronic spin of the NV center is further coupled by hyperfine (HF) interaction to the spin-one ${ }^{14} N$ nucleus, which splits $\omega_{ \pm}$ into three peaks separated by $2.2 \mathrm{MHz}$ [21] (see Supplementary Material). In our experiment, the diamond crystal is glued on top of the chip, and the degeneracy between states $m_{S}= \pm 1$ is lifted with a $B_{N V}=1.4 \mathrm{mT}$ magnetic field applied parallel to the chip and along the $[1,1,1]$ crystalline axis. The NV frequencies being sensitive only to the projection of $B_{N V}$ along the $N-V$ axis, two groups of NVs thus experience different Zeeman effects: those along $[1,1,1]$ (denoted $I$ ) and those along either of the three other $\langle 1,1,1\rangle$ axes (denoted $I I I$ as they are 3 times more numerous). This results in four different ESR frequencies $\omega_{ \pm I, \pm I I I}$.

The qubit $Q$ is a Cooper-pair box of the transmon type [5, 7] with transition frequency $\omega_{Q}$ between its ground state $|g\rangle$ and excited state $|e\rangle$. It is coupled to a nonlinear resonator $R$ which is used to read-out its state, as in related circuit quantum electrodynamics experiments [22. Single-qubit rotations are realized by applying microwave pulses at $\omega_{Q}$ through $R$. Qubit state readout is performed by measuring the phase of a microwave pulse reflected on $R$, which depends on the qubit state; the probability $P_{e}$ to find the qubit in its excited state is then determined by repeating $\sim 10^{4}$ times the same experimental sequence.

The quantum bus $B$, a superconducting resonator with quality factor $\sim 10^{4}$, is electrostatically coupled to the qubit and magnetically coupled to the spin ensemble. In order to bridge the difference in frequency between $Q$ and $N V$, the bus frequency $\omega_{B}$ can be tuned on a nanosecond time scale [23] by applying current pulses through an on-chip line, inducing 
a magnetic flux $\Phi$ through a SQUID embedded in $B[9]$. More information on the qubit readout and setup can be found in the Supplementary Material.

We first characterize our hybrid circuit by spectroscopic measurements. The NV frequencies and coupling constants are obtained by measuring the microwave transmission $\left|S_{21}(\omega)\right|$ through the bus, while scanning its frequency $\omega_{B}(\Phi)$ across the NV resonance. Vacuum Rabi splittings are observed when $\omega_{B}$ matches the spin resonance frequency at $\omega_{+I} / 2 \pi=2.91 \mathrm{GHz}, \omega_{-I} / 2 \pi=2.84 \mathrm{GHz}, \omega_{+I I I} / 2 \pi=2.89 \mathrm{GHz}$, and $\omega_{-I I I} / 2 \pi=2.865 \mathrm{GHz}$ (see Fig. 1). From the data we extract the coupling constants $g_{ \pm I} / 2 \pi=2.9 \mathrm{MHz}$ and $g_{ \pm I I I} / 2 \pi=3.8 \mathrm{MHz}$, the difference between the two values resulting essentially from the larger number of NV centers in group III. Qubit spectroscopy is performed by scanning the frequency of a microwave pulse applied through $R$, and by measuring $P_{e}$, which yields $\omega_{Q} / 2 \pi=2.607 \mathrm{GHz}$. This spectroscopy, measured while scanning $\omega_{B}$ across $\omega_{Q}$, shows an anticrossing (see Fig. 1 1 ) that yields the coupling constant $g_{Q} / 2 \pi=7.2 \mathrm{MHz}$ between $Q$ and $B$.

Throughout the experiments reported in the following, the spins and qubit frequencies are kept fixed, and only $\omega_{B}$ is varied in order to transfer coherently quantum information between $Q$ and $N V$. For this purpose, a key operation is the qubit-bus SWAP gate that transfers an arbitrary qubit state $\alpha|g\rangle+\beta|e\rangle$ into the corresponding photonic state $\alpha|0\rangle_{B}+\beta|1\rangle_{B}$ of the bus, leaving the qubit in $|g\rangle$. This SWAP gate could be performed by tuning $\omega_{B}$ in resonance with $\omega_{Q}$ for a duration $\pi / 2 g_{Q}$ [24]. Here we prefer instead to adiabatically sweep $\omega_{B}$ across $\omega_{Q}$ as this sequence is more immune to flux noise in the SQUID loop of $B$ (see Supplementary Material). This adiabatic SWAP ( $a S W A P$ ) achieves the same quantum operation as the resonant SWAP except for an irrelevant dynamical phase. The experiments then proceed by combining single-qubit rotations, $a S W A P$ gates, and flux pulses placing $B$ and $N V$ in and out of resonance for properly chosen interaction times.

We apply such a sequence with the qubit initially prepared in $|e\rangle$ (see Fig. 2). A first $a S W A P$ converts $|e\rangle$ into the bus Fock state $|1\rangle_{B} ; B$ is brought in or near resonance with the spin ensemble for a duration $\tau$; the resulting $B$ state is then transferred back into the qubit, which is finally read-out. Figure $2 \mathrm{~b}$ shows the resulting curve $P_{e}(\tau)$ when the bus is brought in resonance either with $\omega_{-I I I}$ or $\omega_{-I}$. An oscillation in $P_{e}$ is observed, revealing a storage in the spin ensemble of the single quantum of excitation initially in the qubit at $\tau_{s, I I I}=65 \mathrm{~ns}$ or $\tau_{s, I}=97 \mathrm{~ns}$, and a retrieval back into the qubit at $\tau_{r, I I I}=116 \mathrm{~ns}$ or $\tau_{r, I}=146 \mathrm{~ns}$. The 

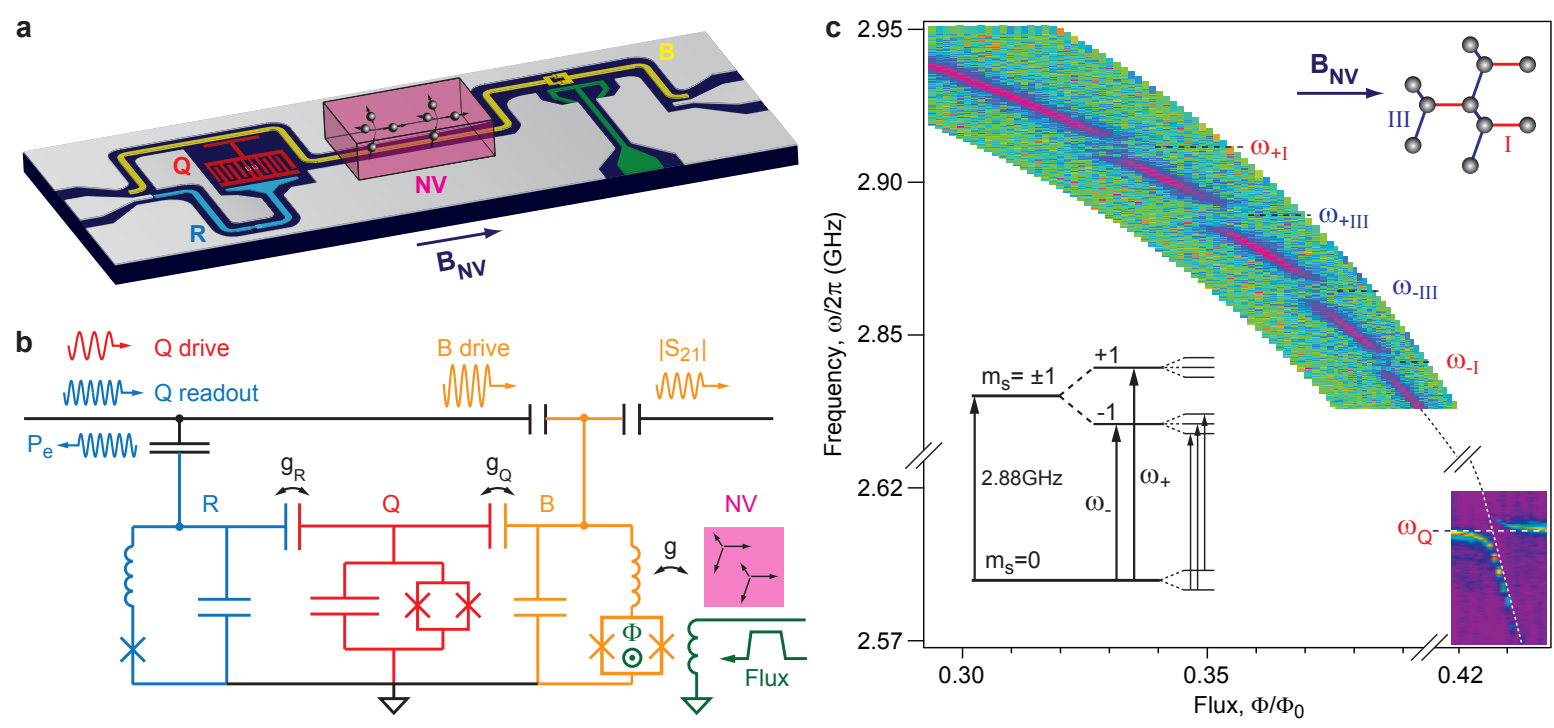

Figure 1: Description of the hybrid quantum circuit demonstrated in this work. a,b Threedimensional sketch of the device and corresponding electrical scheme. The ensemble $N V$ of electronic spins (magenta) consists of $10^{12} \mathrm{NV}$ centers in a diamond crystal glued on the chip surface. The transmon qubit $Q$ (in red) is capacitively coupled to a resonator $R$ (in blue) made nonlinear with a Josephson junction and used to read-out its state. The bus $B$ (in yellow) is electrostatically coupled to $Q$ and magnetically coupled to $N V$. $B$ contains a SQUID that makes its frequency $\omega_{B}(\Phi)$ tunable by changing the flux $\Phi$ in the SQUID loop applied via a fast on-chip current line (in green). A magnetic field $B_{N V}$ is applied parallel to the $[1,1,1]$ crystallographic axis. c, (lower left inset) Energy level structure of NV centers. Transitions between $m_{S}=0$ and $m_{S}= \pm 1$ at frequency $\omega_{ \pm}$are further split in three resonance lines due to the hyperfine interaction with the ${ }^{14} N$ nuclear spin [21]. (main panel) Two-dimensional plot of the transmission $\left|S_{21}\right|(\omega, \Phi)$ through $B$ in $\mathrm{dB}$ units, with $\Phi$ expressed in units of the superconducting flux quantum $\Phi_{0}=h / 2 e$, for a field $B_{N V}=1.4 \mathrm{mT}$ applied to the spins. Color scale goes from $-55 \mathrm{~dB}$ (green) to $-30 \mathrm{~dB}$ (magenta). Four vacuum Rabi splittings are observed whenever $\omega_{B}$ matches one NV center resonance frequency. The four frequencies correspond to the $\omega_{ \pm I, I I I}$ transition frequencies of one of two distinct families of NV centers, being either along the $[1,1,1]$ crystal direction parallel to $B_{N V}$ ( $I$, in red), or along one of the three other possible $\langle 1,1,1\rangle$ axes (labelled $I I I$, in blue), as shown in upper right panel. (main panel, bottom right) Qubit excited state probability $P_{e}$ as a function of the frequency of the exciting microwave and $\Phi$. Color scale goes from 0.1 (purple) to 0.3 (yellow). When $\omega_{B}$ matches the qubit frequency $\omega_{Q}=2.607 \mathrm{GHz}$, the qubit spectrum shows an anticrossing demonstrating its coupling to $B$ with constant $g_{Q} / 2 \pi=7.2 \mathrm{MHz}$. 


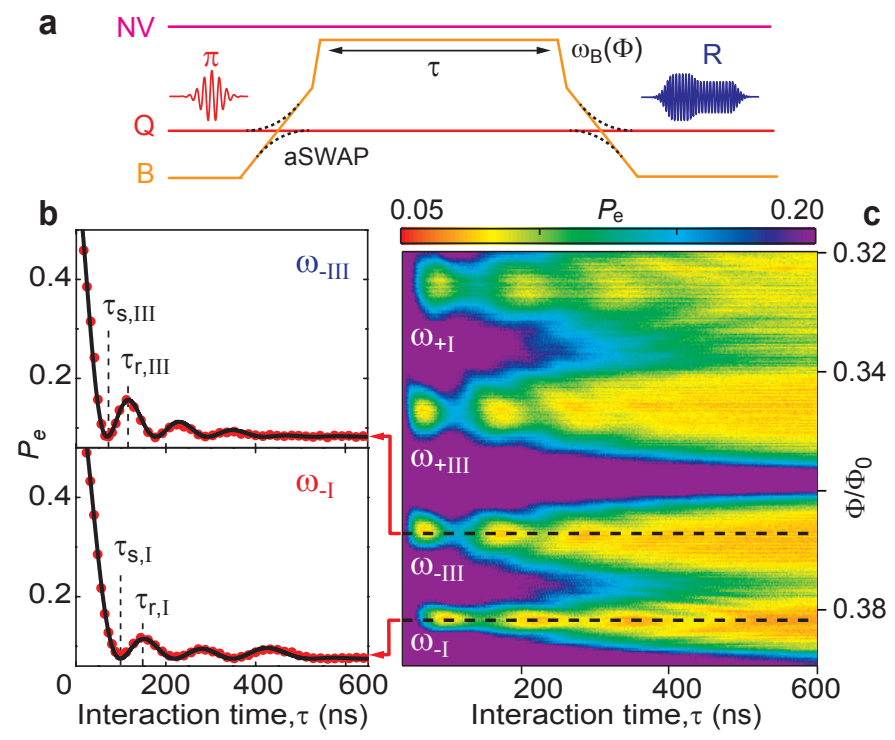

Figure 2: Storage and retrieval of a single quantum of excitation from the qubit to the spin ensemble. a, Experimental sequence showing the microwave pulses used for exciting the qubit in $|e\rangle$ (red) and for reading it out (blue), as well as transition frequencies of the quantum bus (orange), qubit (red), and spins (magenta). b, Experimental (red dots) and theoretical (black line - see text) probability $P_{e}(\tau)$ for $\omega_{B}$ tuned to $\omega_{-I I I}$ (top graph) or $\omega_{-I}$ (bottom graph), showing the storage and retrieval times $\tau_{s}$ and $\tau_{r}$. c. Two-dimensional plot of $P_{e}$ versus interaction time $\tau$ and flux pulse height $\Phi$, showing resonance with the four spin groups. Chevron-like patterns are observed, showing a faster oscillation with reduced amplitude when $\omega_{B}$ is detuned from the spin resonance, as expected. Note that the difference between the $\omega_{-}$and $\omega_{+}$patterns in the same NV group is simply caused by the non-linear dependence of $\omega_{B}$ on $\Phi[9]$.

fidelity of this storage-retrieval process, defined as $P_{e}\left(\tau_{r}\right) / P_{e}(0)$, is 0.14 for group $I I I$ and 0.07 for group $I$. These relatively low values are not due to a short spin dephasing time, but rather to an interference effect caused by the HF structure of NV centers, as evidenced by the non-exponential damping observed in $P_{e}(\tau)$. These measurements are accurately reproduced by a full calculation of the spin-resonator dynamics (see Suppl. Mat. and [20, 25, 26]) taking into account this HF structure, with the linewidth of each HF peak as the only adjustable parameter. A linewidth of $1.6 \mathrm{MHz}$ is in this way determined for the spins in group $I$, and of $2.4 \mathrm{MHz}$ for group $I I I$, this larger value being likely due to a residual misalignment of $B_{N V}$ from the $[1,1,1]$ crystalline axis causing each of the three $<1,1,1>N-V$ orientations non-collinear with the field to experience slightly different Zeeman shifts. We finally note 


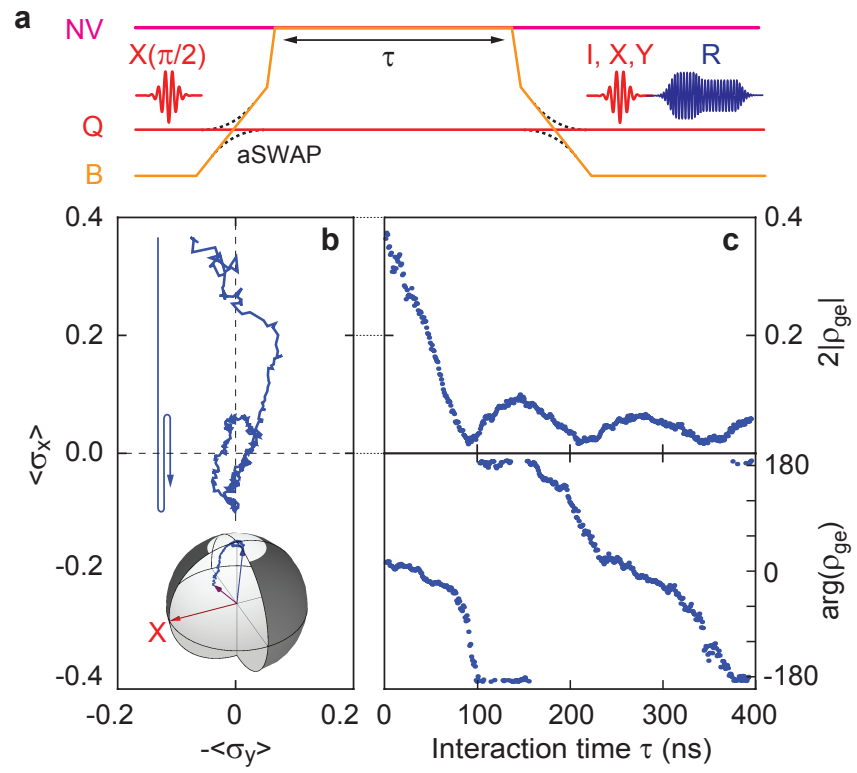

Figure 3: Storage and retrieval of a coherent superposition of states from the qubit to the spin ensemble. a, Experimental pulse sequence: the qubit is prepared by a $\pi / 2$ pulse in state $(|g\rangle+$ $|e\rangle) / \sqrt{2}$, which is transferred to $B$ by an $a S W A P$. $B$ is then immediately tuned to $\omega_{-\mathrm{I}} / 2 \pi=$ $2.84 \mathrm{GHz}$ for an interaction time $\tau$. The quantum state of $B$ is then transferred back to the qubit by an $a S W A P$. Quantum state tomography is finally performed to determine the qubit state by applying either $I, X$, or $Y$ operation to the qubit. b, Trajectory of the qubit Bloch vector on the Bloch sphere (bottom inset), and its projection on the equatorial plane. c, Modulus and phase of the off-diagonal element $\rho_{g e}$ of the qubit density matrix as a function of interaction time $\tau$.

that in both curves shown in Fig. $2 \mathrm{~b} P_{e}(\tau)$ tends towards 0.08 at long times, as is also found with the qubit initially in $|g\rangle$. This proves that the collective spin variable coupled to $B$ is, as requested for experiments in the quantum regime, in its ground state $|0\rangle_{-I,-I I I}$ with a large probability $\sim 0.92$ at equilibrium, which corresponds to a temperature of $\sim 50 \mathrm{mK}$. Varying both $\omega_{B}$ and $\tau$ with the same pulse sequence, we observe similar storage-retrieval cycles at all four spin frequencies (see Fig. 22).

In addition to storing a single excitation from the qubit, one has to test if a coherent superposition of states can be transferred to the spin ensemble and retrieved. For that, we now perform the $a S W A P$ and bring $\omega_{B}$ in resonance with $\omega_{-I}$ after having initialized the qubit in $(|g\rangle+|e\rangle) / \sqrt{2}$ instead of $|e\rangle$, and we reconstruct the Bloch vector of the qubit by quantum state tomography at the end of the sequence. More precisely, we measure $\left\langle\sigma_{X}\right\rangle$, 


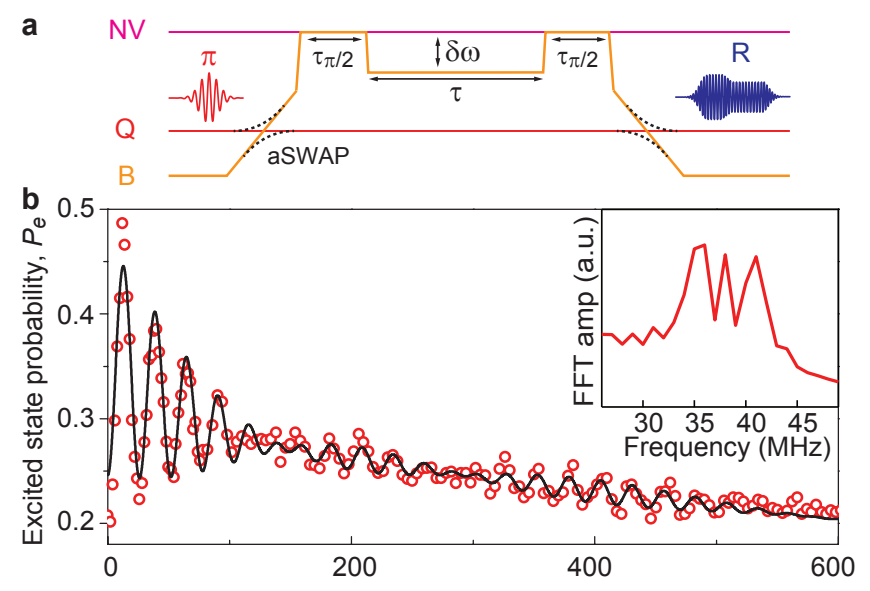

Figure 4: Ramsey-like experiment on the spin ensemble at the single-photon level. a, Experimental pulse sequence: the qubit is prepared in its excited state $|e\rangle$ by a $\pi$ pulse; the state $\left|e, 0_{B}\right\rangle$ is then adiabatically transferred to $\left|g, 1_{B}\right\rangle$ by an $a S W A P$. A fast flux pulse subsequently brings $\omega_{B}$ onto $\omega_{-\mathrm{I}}$, and then lets $B$ and the spins from group $-\mathrm{I}$ interact for half a swap time $\tau_{\mathrm{s}, \mathrm{I}} / 2$, generating an entangled state of the two systems. $B$ is then detuned from the spins by $\delta \omega / 2 \pi=38 \mathrm{MHz}$ during a time $\tau$, and a second half-swap is performed. The quantum state of $B$ is then transferred back to the qubit, which is finally read-out. b, Measured (red circles) and calculated (black line - see text) probability $P_{e}(\tau)$, as well as its Fourier transform (inset) revealing the NV centers HF structure.

$\left\langle\sigma_{Y}\right\rangle$ and $\left\langle\sigma_{Z}\right\rangle$ by using $\pi / 2$ rotations around $Y, X$, or no rotation at all $(I)$ prior to qubit readout. After substracting a trivial rotation around $Z$ occurring at frequency $\left(\omega_{-I}-\omega_{Q}\right)$, we reconstruct the trajectory of this Bloch vector as a function of the interaction time $\tau$. It is plotted in Fig. 3, together with the off-diagonal element $\rho_{g e}$ of the final qubit density matrix, which quantifies its coherence. We find that no coherence is left in the qubit at the end of the sequence for $\tau=\tau_{s, I}$, as expected for a full storage of the initial state into the ensemble. Then, coherence is retrieved at $\tau=\tau_{r, I}$, although with an amplitude $\sim 5$ times smaller than its value at $\tau=0$ (i.e. without interaction with the spins). Note the $\pi$ phase shift occurring after each storage-retrieval cycle, characteristic of $2 \pi$ rotations in the two-level space $\left\{\left|1_{B}, 0_{-I}\right\rangle,\left|0_{B}, 1_{-I}\right\rangle\right\}$. The combination of the results of Figs. 2 and 3 demonstrates that arbitrary superpositions of the two qubit states can be stored and retrieved in a spin ensemble - although with limited fidelity - and thus represents a first proof-of-concept of a spin-based quantum memory for superconducting qubits.

To evaluate the time during which quantum coherence can be stored in the ensemble, 
we perform a Ramsey-like experiment on the spin ensemble at the single-photon level (see Fig. 4): we initially prepare the qubit in $|e\rangle$, transfer its state to $B$, then tune $\omega_{B}$ to $\omega_{-I}$ for a duration $\tau_{\pi / 2}=\tau_{s,-I} / 2$, after which $\omega_{B}$ is suddenly detuned by $\delta \omega / 2 \pi=38 \mathrm{MHz}$ for a time $\tau$. At this point, the joint bus-spin ensemble state is an entangled state $\left(\left|1_{B}, 0_{-I}\right\rangle+e^{i \varphi}\left|0_{B}, 1_{-I}\right\rangle\right) / \sqrt{2}$ with a phase $\varphi=\delta \omega \tau$. $B$ is then put back in resonance with the spins for a second interaction of duration $\tau_{\pi / 2}$ that converts the phase $\varphi$ into population of $\left|1_{B}, 0_{-I}\right\rangle$. This population is finally transferred to the qubit, and read-out. Oscillations at frequency $\delta \omega$ are observed in $P_{e}(\tau)$ as seen in Fig. 4, confirming that the resonator and the spins are entangled after the first $\pi / 2$ pulse. These oscillations are modulated by a beating pattern, with an overall damping of the oscillations envelope in $\sim 200$ ns. Quite remarkably, this beating observed in the qubit excited state probability is directly caused by the HF structure of NV centers, as proved by the Fourier transform of $P_{e}(\tau)$ which shows the three HF lines. The full calculation of the system dynamics quantitatively captures both the beatings and the oscillations damping, which is thus completely explained by the $1.6 \mathrm{MHz}$ inhomogeneous linewidth of each HF line taken into account in the theory.

The previous results suggest that the storage of quantum information in the NV centers ensemble is at present limited both by its HF structure and by the inhomogeneous broadening of its resonance. This broadening is attributed to dipolar interactions between the NV centers and residual paramagnetic impurities (likely neutral nitrogen atoms) in the diamond crystal. Purer crystals could thus greatly improve the present performance of our device. Note that the hyperfine coupling to the nuclear spin of ${ }^{14} N$ could be turned into a useful resource if quantum information was transferred from the electron spin to the nuclear spin degree of freedom, which has much narrower linewidth. Finally refocusing techniques borrowed from quantum memories in the optical domain [27] should also lead to increase in the storage time by two orders of magnitude.

In conclusion our experiments bring the first proof of concept of a spin-based quantum memory for superconducting qubits. In a longer-term perspective, they open the way to the implementation of genuine quantum lab-on-chips, where superconducting qubits would coherently interact with electron and nuclear spins as well as optical photons.

Note: During redaction of this manuscript we became aware of related work demonstrating the coherent dynamics of a flux qubit coupled to an ensemble of NV centers in diamond [28]. 
Acknowledgements We acknowledge useful discussions with K. Moelmer, F. Jelezko, J. Wrachtrup, D. Twitchen, and within the Quantronics group, and technical support from P. Sénat, P.-F. Orfila, T. David, J.-C. Tack, P. Pari, P. Forget, M. de Combarieu. We acknowledge support from European projects Midas and Solid, ANR project Masquelspec, C'Nano, Capes, and Fondation Nanosciences de Grenoble.

\section{Methods Summary}

Superconducting circuit parameters The transmon parameters are measured by standard spectroscopy, yielding a Josephson energy $E_{J} / h=5.2 \mathrm{GHz}$ and a Coulomb energy for a Cooper-pair $E_{C} / h=0.66 \mathrm{GHz}$. Its relaxation time $T_{1}=1.75 \mu \mathrm{s}$ and coherence time $T_{2}=2.2 \mu \mathrm{s}$ at the bias point used in this work were measured by standard pulse sequences.

The bus resonator $B$ could be tuned from a maximum frequency $\omega_{B}(0) / 2 \pi=3.004 \mathrm{GHz}$ down to $2.5 \mathrm{GHz}$. Its quality factor is $2 \cdot 10^{4}$ at $\Phi=0$ in presence of the diamond sample (which thus does not introduce dielectric losses contrary to what was reported in [16, 18]). This quality factor degrades progressively as $\omega_{B}$ is tuned towards lower frequencies. However the bus resonator energy relaxation time $T_{c a v}=1.5 \mu$ s was measured using the qubit as explained in [29], and was found not to depend on the flux bias. This indicates that the quality factor degradation is due to low-frequency noise, likely flux noise in the SQUID loop.

The readout resonator $R$ has a frequency $\omega_{R} / 2 \pi=3.468 \mathrm{GHz}$ and a quality factor $Q=$ 500. Its nonlinearity is brought by a Josephson junction of critical current $650 \mathrm{nA}$ yielding a Kerr constant $K / \omega_{R}=-4.5 \cdot 10^{-6}$ [30]. Readout pulses have a frequency $3.456 \mathrm{GHz}$. The coupling between $Q$ and $R$ is estimated to be $g_{R} / 2 \pi=30 \mathrm{MHz}$.

Theory Each spin is modelled by an effective harmonic oscillator $b_{j}$ of frequency $\omega_{j}$, following the Holstein-Primakoff approximation valid in the low-excitation limit. The qubit, bus resonator, and spin ensemble are described by Hamiltonians $H_{Q} / \hbar=-\left(\omega_{Q} / 2\right) \sigma_{Z}$, $H_{B} / \hbar=\omega_{B}(\Phi) a^{\dagger} a$ and $H_{N V} / \hbar=\sum \omega_{j} b_{j}^{\dagger} b_{j}, \sigma_{Z}$ being the Pauli matrix, $a$ being the bus resonator annihilation operator. Coupling of the qubit to the bus resonator is described by a Jaynes-Cummings Hamiltonian $H_{Q-B} / \hbar=g_{Q}\left(\sigma^{+} a+h . c\right)$ where $\sigma^{+}$is the qubit raising operator. Coupling between the bus resonator and the spin ensemble is described by a TavisCummings Hamiltonian $H_{B-N V} / \hbar=\sum_{j=1}^{N} g_{j}\left(b_{j}^{\dagger} a+h . c.\right)$. This Hamiltonian can be rewritten as $H_{B-N V} / \hbar=\sum_{K=-I I I,-I,+I,+I I I} g_{K}\left(b_{K}^{\dagger} a+h . c.\right)$ where $g_{K}$ represents the collective coupling between $\mathrm{B}$ and each of the four spin groups $( \pm I, \pm I I I)$ and $b_{K}=\left(1 / g_{K}\right) \sum_{j=1}^{N} g_{\mathrm{j}} b_{\mathrm{j}}$ is the collective excitation annihilation operator. Excited states of the spin ensemble are 
defined for each family by applying collective operators $b_{K}$ and $b_{K}^{\dagger}$ to the ground state $\left|0_{K}\right\rangle$ (for instance $\left|1_{-I}\right\rangle=b_{-I}^{\dagger}\left|0_{-I}\right\rangle$ ). The spin-resonator dynamics can be calculated from this model, as explained in [25, 26] and in the Supplementary Material.

Adiabatic pulse parameters Our adiabatic SWAP operation proceeds as follows: $\omega_{B}$ starts at $2.52 \mathrm{GHz}$, is first ramped up to $2.589 \mathrm{GHz}$ in $60 \mathrm{~ns}$, then to $2.643 \mathrm{GHz}$ in $350 \mathrm{~ns}$, then to $2.687 \mathrm{GHz}$ in $40 \mathrm{~ns}$. See the Supplementary Information for more details on the pulse optimization.

[1] Roos, C.F. et al., Bell States of Atoms with Ultralong Lifetimes and Their Tomographic State Analysis, Phys. Rev. Lett. 92, 220402 (2004).

[2] Balasubramanian, G. et al., Ultralong spin coherence time in isotopically engineered diamond, Nature Materials 8, 383 (2009).

[3] DiCarlo, L. et al., Preparation and measurement of three-qubit entanglement in a superconducting circuit, Nature 467, 574-578 (2010).

[4] Neeley, M. et al., Generation of three-qubit entangled states using superconducting phase qubits, Nature 467, 570-574 (2010).

[5] Schreier, J. A. et al., Suppressing charge noise decoherence in superconducting charge qubits, Phys. Rev. B 77, 180502 (2008).

[6] Paik, H. et al., How coherent are Josephson junctions?, arxiv:cond-mat/1105.4652 (2011).

[7] Koch, J. et al., Charge-insensitive qubit design derived from the Cooper pair box, Phys. Rev. A 76, 042319 (2007).

[8] Jelezko, F. et al., Observation of Coherent Oscillations in a Single Electron Spin, Phys. Rev. Lett. 92, 076401 (2004).

[9] Palacios-Laloy, A. et al., Tunable Resonators for Quantum Circuits, J. Low Temp. Phys. 151, 1034 (2008).

[10] Imamoglu, A., Cavity QED Based on Collective Magnetic Dipole Coupling: Spin Ensembles as Hybrid Two-Level Systems, Phys. Rev. Lett. 102, 083602 (2009).

[11] Wesenberg, J.H. et al., Quantum Computing with an Electron Spin Ensemble, Phys. Rev. Lett. 103, 070502 (2009).

[12] D. Marcos et al., Coupling Nitrogen-Vacancy Centers in Diamond to Superconducting Flux 
Qubits, Phys. Rev. Lett. 105, 210501 (2010).

[13] Wallraff, A. et al., Strong coupling of a single photon to a superconducting qubit using circuit quantum electrodynamics, Nature 431, 162-167 (2004).

[14] O'Connell, A.D. et al., Quantum ground state and single-phonon control of a mechanical resonator, Nature 464, 697-701 (2010).

[15] Neeley, M. et al., Process tomography of quantum memory in a Josephson-phase qubit coupled to a two-level state, Nature Phys. 4, 523-526 (2008).

[16] Kubo, Y. et al., Strong Coupling of a Spin Ensemble to a Superconducting Resonator, Phys. Rev. Lett. 105, 140502 (2010).

[17] Schuster, D.I. et al., High-Cooperativity Coupling of Electron-Spin Ensembles to Superconducting Cavities, Phys. Rev. Lett. 105, 140501 (2010).

[18] Amsüss, R. et al., Cavity QED with Magnetically Coupled Collective Spin States, Phys. Rev. Lett. 107, 060502 (2011).

[19] Wu, H. et al., Storage of Multiple Coherent Microwave Excitations in an Electron Spin Ensemble, Phys. Rev. Lett. 105, 140503 (2011).

[20] Kubo, Y. et al., Storage and Retrieval of a Microwave Field in a Spin Ensemble, arxiv:condmat/1109.3960

[21] Felton, S., et al., Hyperfine interaction in the ground state of the negatively charged nitrogen vacancy center in diamond, Phys. Rev. B 79, 075203 (2009).

[22] Mallet, F. et al., Single-shot qubit readout in circuit quantum electrodynamics, Nature Phys. 5, 791 (2009).

[23] Sandberg, M. et al., Tuning the field in a microwave resonator faster than the photon lifetime, Appl. Phys. Lett. 92, 203501 (2008).

[24] Hofheinz, M. et al., Generation of Fock states in a superconducting quantum circuit, Nature 454, 310 (2008).

[25] Diniz, I. et al., Strongly coupling a cavity to inhomogeneous ensembles of emitters : potential for long lived solid-state quantum memories, arxiv:quant-ph/1101.1842 (2011).

[26] Kurucz, Z., Wesenberg, J.H., and Molmer, K., Spectroscopic properties of inhomogeneously broadened spin ensembles in a cavity, Phys. Rev. A 83, 053852 (2011).

[27] Lvovsky, A.I., Sanders, B.C., and Tittel, W., Optical quantum memory, Nature Phot. 3, 706 (2009). 
[28] Zhu, X. et al., Coherent coupling of a superconducting flux-qubit to an electron spin ensemble in diamond, to appear in Nature (2011).

[29] Wang, H. et al., Measurement of the Decay of Fock States in a Superconducting Quantum Circuit, Phys. Rev. Lett. 101, 240401 (2008).

[30] Ong, F. et al., Circuit QED with a Nonlinear Resonator: ac-Stark Shift and Dephasing, Phys. Rev. Lett. 106, 167102 (2011). 


\section{Supplementary information for "Hybrid quantum circuit with a superconducting}

qubit coupled to a spin ensemble"

\section{A. Diamond sample preparation and characterization}

The sample we use is a polished (110) plate of dimensions $2.2 \times 1 \times 0.5 \mathrm{~mm}^{3}$ taken from a type-Ib HPHT crystal which contained 40 ppm of neutral substitutional nitrogen (the P1 centers) as measured by IR absorption. It has been irradiated by $2 \mathrm{MeV}$ electrons at $700^{\circ} \mathrm{C}$ with a total dose of $6.4 \times 10^{18} \mathrm{e} / \mathrm{cm}^{2}$ and annealed at $1000^{\circ} \mathrm{C}$ for 2 hours in vacuum. The high temperature irradiation was employed to minimize the residual unwanted defects.

The resulting concentration of negatively-charged NV centers was measured by comparing the sample photoluminescence (PL) to the one obtained from a single NV center. For that purpose, a continuous laser source operating at $532 \mathrm{~nm}$ wavelength was tightly focused on the sample through a high numerical aperture oil-immersion microscope objective. The NV center PL was collected by the same objective, spectrally filtered from the remaining pump light and directed to a silicon avalanche photodiode. After calibration of the PL response associated with a single NV center, a PL raster scan of the sample directly indicates the NV center content since the excitation volume is known. As shown in Fig. 5(a), the NV center concentration is found rather inhomogeneous over the sample, with an average density of $4.4 \times 10^{17} \mathrm{~cm}^{-3}$. Such a value is in good agreement with the spin ensemble-resonator coupling constants reported in the main text of the manuscript. 

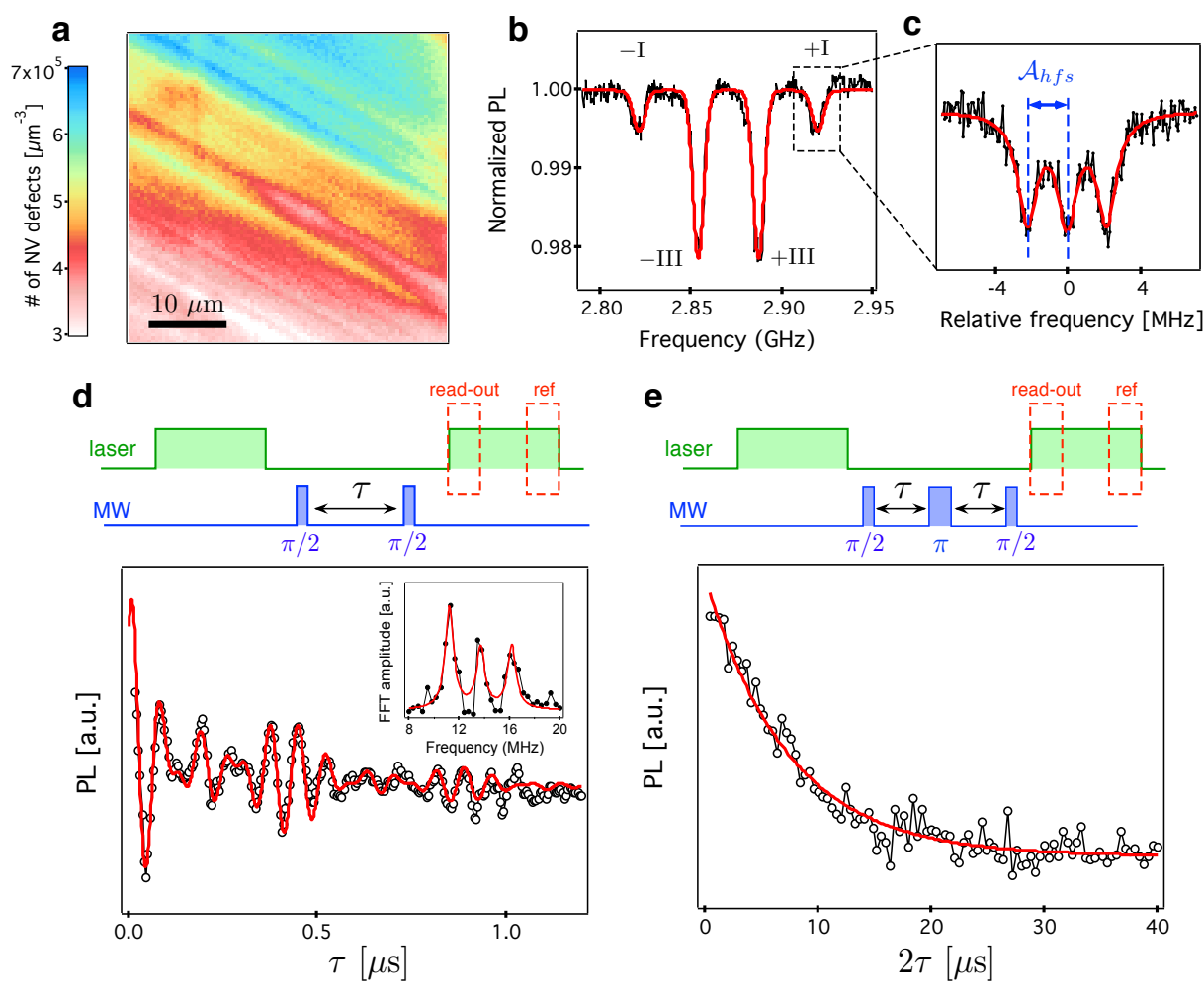

Figure 5: Characterization of the diamond sample at room temperature. a,Twodimensional map of the NV center density for a depth of $5 \mu \mathrm{m}$ inside the diamond sample. $\mathbf{b}$, Optically detected ESR spectrum with a static magnetic field $B \approx 1.5 \mathrm{mT}$ applied along the [111] axis of the diamond crystal. Four ESR lines are observed, corresponding to $m_{s}=0 \rightarrow m_{s}= \pm 1$ spin transitions for the two subsets of NV centers crystalline orientations $( \pm \mathrm{III}$ and $\pm \mathrm{I})$. c, Hyperfine structure. Data fitting with Lorentzian functions leads to $\mathcal{A}_{h f}=2.17 \pm 0.02 \mathrm{MHz}$ as expected from hyperfine interaction with ${ }^{14} \mathrm{~N}$ nuclear spins. d, Ramsey fringes recorded for the subset of [111]-oriented NV centers with a microwave detuning $\delta=13 \mathrm{MHz}$ from the ESR line at $\omega_{+I} / 2 \pi=2.915 \mathrm{GHz}$. The laser pulses used in the Ramsey sequence have a duration of $30 \mu$ s and the laser power is set at $40 \mathrm{~mW}$. For data analysis, the NV center PL recorded during the first $10 \mu \mathrm{s}$ of the laser pulses is used for spin-state read-out while the PL recorded during the last $10 \mu \mathrm{s}$ is used as reference. The red solid line is data fitting with the function $\exp \left[-\tau / T_{2}^{*}\right] \times \sum_{i=-1}^{1} \cos \left[2 \pi\left(\delta+i \mathcal{A}_{h f}\right) \tau\right]$. The inset shows the Fourier-transform of the free induction decay. Solid lines are data fitting with Lorentzian functions. e, Measurement of the coherence time $T_{2}$ for the subset of [111]-oriented NV centers using a $\pi / 2-\tau-\pi-\tau-\pi / 2$ spin echo sequence. Data fitting with an exponential decay leads to $T_{2}=7.3 \pm 0.4 \mu \mathrm{s}$. 
Electron spin resonance (ESR) measurements were performed at room temperature by applying a microwave field through a copper microwire directly spanned on the diamond surface. In addition, a static magnetic field $B \approx 1.5 \mathrm{mT}$ was applied along the [111] axis of the diamond crystal. As explained in the main text, such a magnetic field orientation allows both to lift the degeneracy of $m_{s}= \pm 1$ spin sublevels and to divide the NV center ensemble into two sub-groups of crystallographic orientations which experience different Zeeman splittings. Optically detected ESR spectra were recorded by sweeping the frequency of the microwave field while monitoring the PL intensity. As shown in Fig. 5(b), when the microwave frequency is resonant with a transition linking $m_{s}=0$ and $m_{s}= \pm 1$ spin sublevels, ESR is evidenced as a dip of the PL signal owing to spin-dependent PL response of the NV center [1, 2]. In the following, we focus the study on the ESR line at $\omega_{+I} / 2 \pi=$ $2.915 \mathrm{GHz}$, which corresponds to the $m_{s}=0 \rightarrow m_{s}=+1$ spin transition for the subset of [111]-oriented NV centers. The nitrogen atom of NV centers in our sample being a ${ }^{14} \mathrm{~N}$ isotope (99.6\% abundance), corresponding to a nuclear spin $I=1$, each electron spin state is further split into three sublevels by hyperfine interaction with a splitting $\mathcal{A}_{h f}=-2.16$ $\mathrm{MHz}$ between ESR frequencies associated with different nuclear spin projections [6]. This hyperfine structure can be easily observed in our sample by decreasing the microwave power in order to reduce power broadening of the ESR linewidth [3] (Fig. 5(c)).

To probe coherence properties of this subset of NV centers, Ramsey fringes were first recorded by using the usual sequence consisting of two microwave $\pi / 2$-pulses separated by a variable free evolution duration $\tau$ (Fig. $5(\mathrm{~d})$ ) [4, 5]. The free induction decay signal exhibits beating frequencies which correspond to the hyperfine components of the NV center. Data fitting of the free induction decay signal leads to a dephasing time $T_{2}^{*}=390 \pm 30$ ns of the NV center electron spins and its Fourier transform spectrum reveals the ${ }^{14} \mathrm{~N}$ hyperfine structure with a linewidth (FWHM) $\Gamma=810 \pm 90 \mathrm{kHz}$ for each peak (see inset of Fig. 5(d)).

The dephasing time can be greatly enhanced by decoupling the electron spin from its local environment with a spin echo sequence (Fig. 5(e)). Using this technique, the dephasing time of the NV center ensemble reaches $T_{2}=7.3 \pm 0.4 \mu \mathrm{s}$ at room temperature. At high spin densities, this quantity is limited by the interaction with a bath of paramagnetic impurities including NV centers themselves and P1 centers [7, 8]. 


\section{B. Superconducting circuit fabrication and measurement setup}

The superconducting circuit is fabricated on a silicon chip oxidized over $50 \mathrm{~nm}$. A $150 \mathrm{~nm}$ thick niobium layer is first deposited by magnetron sputtering and then dry-etched in a $S F_{6}$ plasma to pattern the readout resonator $R$, the bus resonator $B$, the current lines for frequency tuning, and the input waveguides. Finally, the transmon qubit $Q$, the coupling capacitance between $Q$ and $B$, the Josephson junction of $R$, the SQUID in $B$, are fabricated by double-angle evaporation of aluminum through a shadow mask patterned using e-beam lithography. The first layer of aluminum is oxidized in a $\mathrm{Ar}-\mathrm{O}_{2}$ mixture to form the oxide barrier of the junctions. The chip is glued with wax on a printed circuit board (PCB) and wire bonded to it. The PCB is then screwed in a copper box anchored to the cold plate of a cryogen-free dilution refrigerator. A complete scheme of the measurement setup and fridge wiring is shown in Fig. 6 .

\section{Qubit readout}

The qubit readout method we use is explained in detail in [9]. It relies on the nonlinearity of the readout resonator $R$ operated in the so-called JBA mode where it behaves as a sampleand-hold detector. More precisely, we apply a readout pulse of frequency $\omega / 2 \pi=3.456 \mathrm{GHz}$ slightly lower than the resonance frequency $\omega_{R} / 2 \pi=3.468 \mathrm{GHz}$, and of power $P_{R}$ chosen so that the resonator is driven close to its bistability, in a regime where the field inside the resonator can switch from a low-amplitude state $L$ to a high-amplitude state $H$. This switching is easily detected by measuring the phase of the reflected readout pulse. Repeating the same sequence then yields the resonator switching probability $P_{s w}$ for a given readout pulse power. This allows to reconstruct so-called S-curves $P_{s w}\left(P_{R}\right)$ which change from 0 to 1 in a narrow power range, close to bistability (see Fig. 7). Due to the qubit-resonator dispersive coupling, the readout resonator $R$ frequency is shifted by a qubit-state-dependent quantity $\pm \chi$ so that for well-chosen pulse frequency and power the qubit state is mapped onto the resonator dynamical state at the end of the readout pulse (see Fig. 7). The measured switching probability $P_{s w}$ is therefore directly linked to the qubit excited state probability $P_{e}$ which is the quantity of interest in our experiment.

That $P_{e}$ is not directly given by $P_{s w}$ is due to readout errors caused either by a too small 

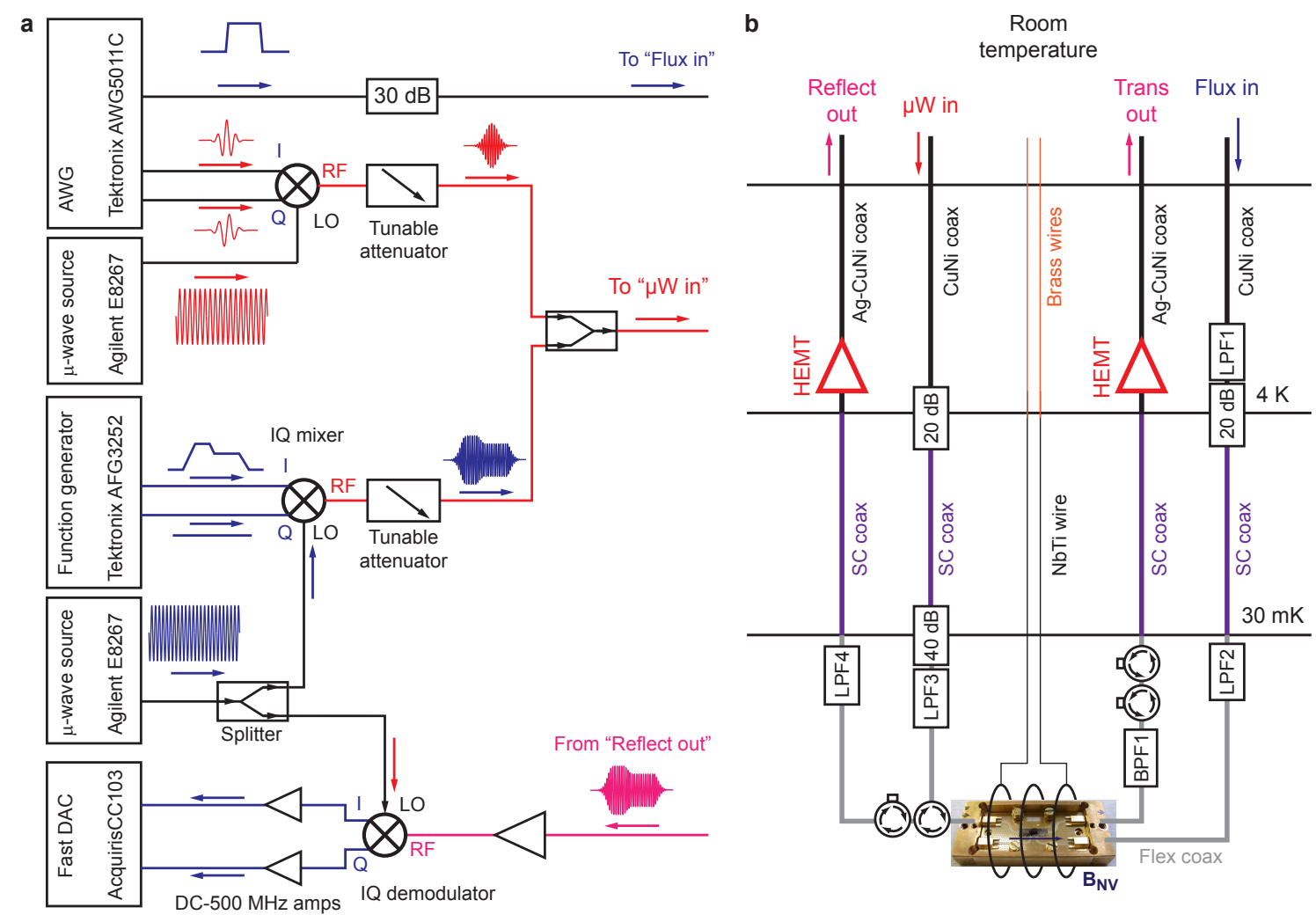

Figure 6: Measurement setup and wiring. a, Full configuration of the measurement apparatus at room temperature. b, Scheme of the wiring inside the dilution refrigerator. LPF1, LPF2, LPF3, and LPF4 are low-pass filters with cutoff frequencies $1.35 \mathrm{GHz}, 450 \mathrm{MHz}, 5.4 \mathrm{GHz}$, and 5.4 $\mathrm{GHz}$, respectively. BPF1 is a band-pass filter with a bandwidth of $2.5-4 \mathrm{GHz}$. CuNi coax is a coaxial cable made of $\mathrm{CuNi}$, and $\mathrm{Ag}-\mathrm{CuNi}$ coax is a silver-plated $\mathrm{CuNi}$ coaxial cable. $\mathrm{SC}$ coax is a superconducting NbTi coaxial cable. Flex coax is a low-loss flexible coaxial cable. Rectangles represent ports terminated by $50 \Omega$. The cryogenic microwave amplifier is a CITCRYO $1-12$ from Caltech, with gain $\sim 38 \mathrm{~dB}$ and noise temperature $\sim 5 \mathrm{~K}$ at $3 \mathrm{GHz}$. A DC magnetic field $B_{N V}$ is applied parallel to the chip by passing a DC current through an outer superconducting coil. The sample box and the coil are surrounded by two magnetic shieldings consisting of a lead cylinder around which permalloy tape is wrapped. The sample box, coil, and the shieldings are thermally anchored at the mixing chamber.

$\chi$ or by qubit relaxation between the end of the experimental sequence and the time at which readout effectively takes place. These errors can be modelled with two parameters : the probability $e_{0}$ that the resonator switches despite the qubit being in $|g\rangle$ at the end of 


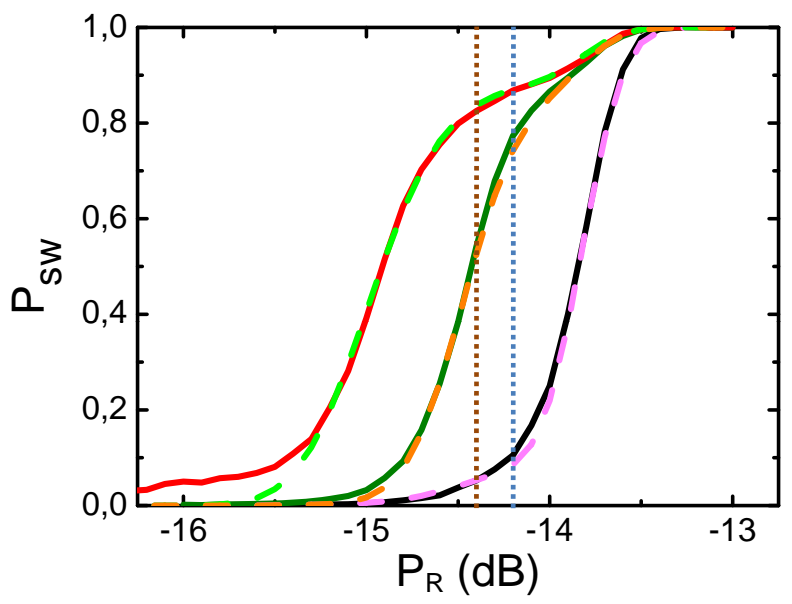

Figure 7: Readout resonator switching probability $P_{s w}$ as a function of readout pulse power $P_{R}$ (S-curves). Black solid line : S-curve with the qubit in thermal equilibrium. Olive solid line: Scurve with the qubit prepared in $|e\rangle$ by a $\pi$ pulse just before the readout. Red solid line: S-curve with the qubit prepared in $|e\rangle$ by a $\pi$ pulse, measured with a composite readout pulse including a $\pi$ pulse on the $|e\rangle-|f\rangle$ transition followed by the usual readout pulse. Dashed lines represent fits of these S-curves using a sum of three Erf functions (corresponding to the three transmon states $|g\rangle,|e\rangle,|f\rangle)$ with different weights. In this way the thermal population of the qubit $P_{e, e q}=0.08$ is evaluated as explained in the text. Note that this thermal excitation is responsible for the "foot" of the black curve. Dotted blue vertical line indicates the readout power used for simple readout pulses, dotted brown vertical line indicates the readout power used for the composite readout pulse method.

the experimental sequence, and the probability $e_{1}$ that the resonator doesn't switch while the qubit is in $|e\rangle$. In order to determine $e_{0}$ and $e_{1}$, we measure the switching probability $P_{s w 0}$ for a qubit at thermal equilibrium, and after a $\pi$ pulse $P_{s w \pi}$ that we assume ideal in the sense that it swaps states $|g\rangle$ and $|e\rangle$ with $100 \%$ efficiency. An additional complication arises from the fact that the qubit has a small but finite probability $P_{e, e q}$ to be found in $|e\rangle$ even at thermal equilibrium, due to the rather low qubit frequency chosen in the experiment to match the NV centers. We therefore first estimate $P_{e, e q}$ by fitting the shape of S-curves at equilibrium and after a $\pi$ pulse to a simple model, yielding $P_{e, e q}=0.08$ in our experiment (see Fig. 7). This corresponds to an effective electromagnetic temperature of $50 \mathrm{mK}$, slightly higher than the cryostat base temperature $30 \mathrm{mK}$ possibly due to imperfect filtering of the flux lines. We then find $e_{0}$ and $e_{1}$ by solving the system of two equations 


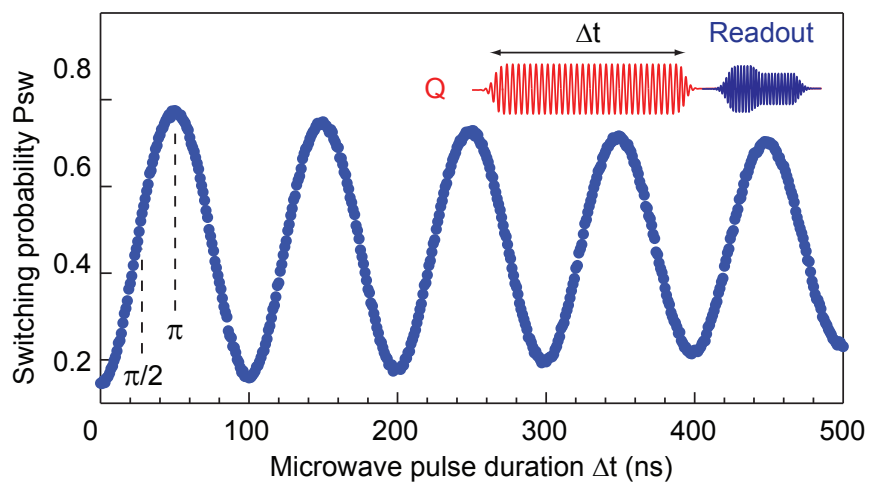

Figure 8: Rabi oscillations. The switching probability $P_{S W}$ of readout resonator $R$ is plotted as a function of microwave pulse duration $\Delta t$. Inset: pulse sequence used in this measurement. Each data point of $P_{S W}$ was constructed by repeating this sequence $10^{4}$ times.

$P_{s w 0}=e_{0}\left(1-P_{e, e q}\right)+\left(1-e_{1}\right) P_{e, e q}$ and $P_{s w \pi}=e_{0} P_{e, e q}+\left(1-e_{1}\right)\left(1-P_{e, e q}\right)$. This allows to determine $P_{e}$ from the directly measured $P_{s w}$ since $P_{s w}=e_{0}\left(1-P_{e}\right)+\left(1-e_{1}\right) P_{e}$.

An additional complication arises from the fact that the fidelity of the readout can be enhanced (i.e. $e_{0}$ and $e_{1}$ lowered) by using the second excited state $|f\rangle$ of the transmon: for that, one applies a $\pi$ pulse on the $|e\rangle-|f\rangle$ transition just prior to readout, resulting in a so-called composite readout pulse. As explained in [9] this reduces readout errors caused by relaxation during the readout pule. Due to technical complications, we use the composite readout pulse method only in experiments reported in figures 2 and 4 a of the main article. The other experiments were performed with simple readout pulses. As a result two different sets of errors $e_{0}$ and $e_{1}$ were determined for each of the two types of readout pulses. For composite readout pulses, we find $e_{0}=0$ and $e_{0}=0.1$, indicating a very high fidelity readout consistent with [9]. Without the composite readout pulse we find $e_{0}=0$ and $e_{0}=0.33$. From these values we convert the measured $P_{s w}$ into $P_{e}$ in all our experiments.

\section{Qubit state manipulation}

Single-qubit operations are carried out by applying Gaussian shaped microwave pulses 10] at $\omega_{Q}$. These pulses are generated as explained above by mixing a CW source at $\omega_{Q}-\omega_{S}$ with a Gaussian shaped pulse modulated at $\omega_{S}$ using an IQ mixer. Before the measurement such as shown in the main text, the system was calibrated to compensate the mixer imperfections 

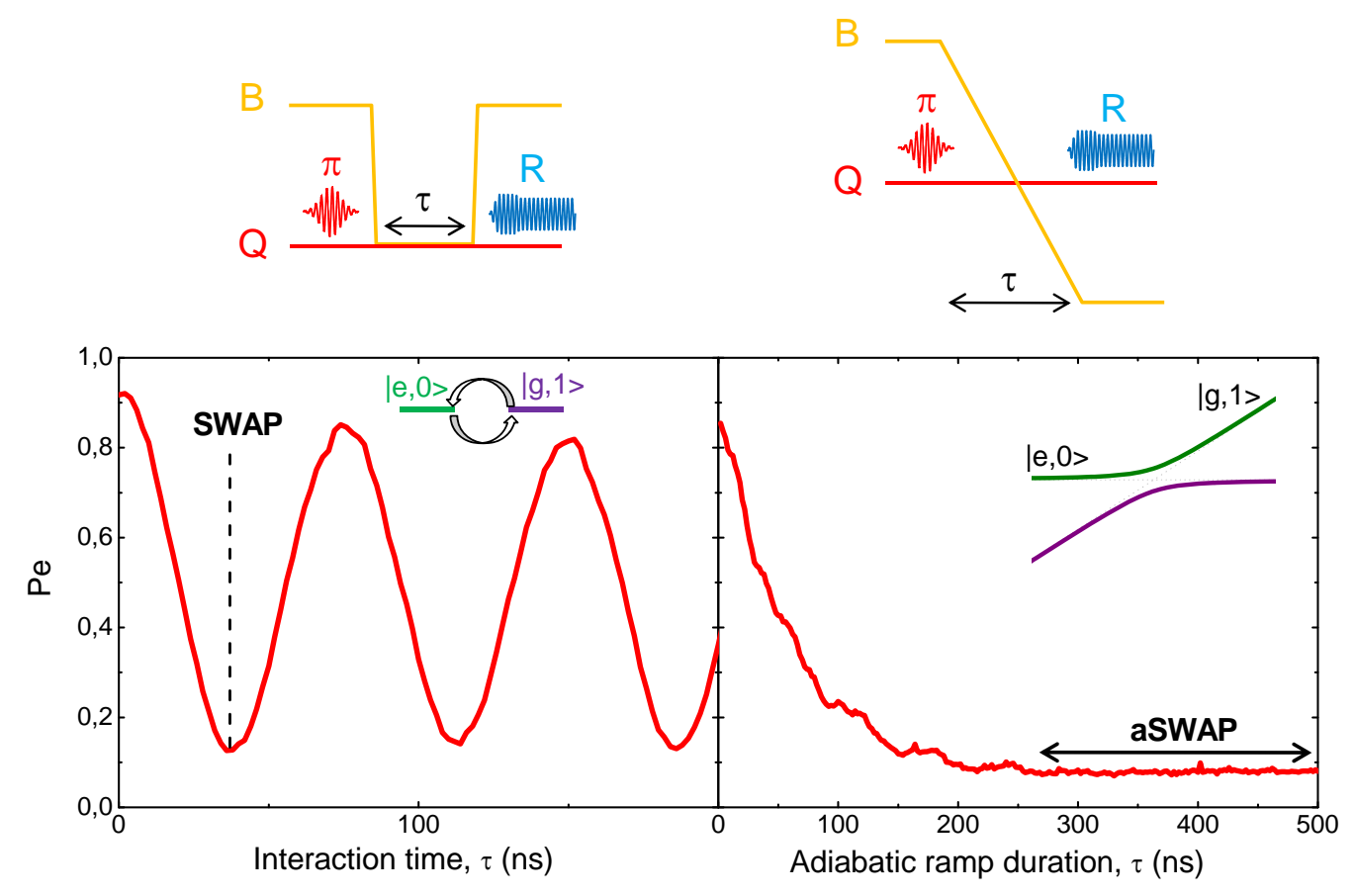

Figure 9: Comparison between resonant and adiabatic SWAP pulses. Left panel: principle of a resonant SWAP. After excitation of the qubit in $|e\rangle, B$ is put suddenly into resonance with $Q$ for an interaction time $\tau$ during which $|e, 0\rangle$ and $|g, 1\rangle$ exchange periodically energy. After a time $\tau=37 \mathrm{~ns}$, the qubit excitation is transferred to $B$. Right panel: principle of an adiabatic SWAP (aSWAP). The qubit is excited in $|e\rangle$, after what $\omega_{B}$ is ramped through $\omega_{Q}$ in a time $\tau$, and the state of $Q$ is finally read-out. For long enough ramp durations (for this sequence $\tau \gtrsim 300 \mathrm{~ns}$ ), the qubit excited state population is fully transferred into the bus.

(amplitude and phase imbalance, offsets). By changing the sideband frequency $\omega_{S}$ it is also possible to apply pulses on the $|e\rangle-|f\rangle$ transition as requested sometimes for readout. Resulting Rabi oscillations are shown in 8 . Here the $\pi$ pulse and $\pi / 2$ pulse are defined to be $50 \mathrm{~ns}$ and $25 \mathrm{~ns}$ respectively.

\section{E. Adiabatic SWAP pulses}

SWAP operations between the qubit and the resonator quantum bus can be performed by tuning suddenly $\omega_{B}$ in resonance with $\omega_{Q}$ for a duration $\pi / 2 g_{Q}$. We show in Fig. 9 the resulting vacuum Rabi oscillations. In the experiment however, we found out that such a resonant SWAP operation was not stable enough to allow subsequent data acquisition longer 
than $\sim 15$ minutes. The problem is caused by flux noise in the SQUID loop which causes $\omega_{B}(\Phi)$ to change over time so that the amplitude of flux pulse needed to perform the vacuum Rabi oscillations in resonance also changes in time. We note that we found a much larger flux noise for $B_{N V}=1.4 \mathrm{mT}$ than $B_{N V}=0 \mathrm{mT}$, probably due to vortices being trapped in the superconducting thin films around the SQUID.

To circumvent this problem, we resort instead to adiabatic SWAP operations in which $\omega_{B}$ is adiabatically ramped through resonance with $\omega_{Q}$ so that state $|e, 0\rangle$ is adiabatically converted into $|g, 1\rangle$ for a sufficiently slow flux pulse, yielding the same operation as the resonant SWAP (see Fig. 9). Finding good parameters for the pulse requires some optimization since a too fast pulse will not be adiabatic while a too slow pulse will strongly reduce the signal because of energy relaxation either in the qubit or in the resonator bus. The final parameters that we used are: $\omega_{B}$ starts at $2.52 \mathrm{GHz}$, is first ramped up to $2.589 \mathrm{GHz}$ in $60 \mathrm{~ns}$, then to $2.643 \mathrm{GHz}$ in $350 \mathrm{~ns}$, then to $2.687 \mathrm{GHz}$ in $40 \mathrm{~ns}$ (with a qubit frequency $\left.\omega_{Q} / 2 \pi=2.607 \mathrm{GHz}\right)$.

\section{F. Theory}

We now explain in more detail how the theory curves in figures 2 and 4 of the article are calculated. The calculation assumes that the qubit state is perfectly transferred to the resonator bus $B$, so that the measured $P_{e}$ perfectly maps the final resonator population in the $\left|1_{B}\right\rangle$ state. Each result of the calculation is rescaled in amplitude and offset to match the experimental data (this accounts for the additional losses caused by relaxation of the qubit or resonator during the pulse sequence, and in particular during the two $a S W A P \mathrm{~s})$. Apart from that, all the calculations are performed using the following theory, and one unique parameters set for the whole paper.

\section{Rabi oscillations (figure 2)}

What needs to be calculated is the final probability to find a photon in the bus resonator after the resonator-spins interaction, assuming the resonator is in $\left|1_{B}\right\rangle$ at time $\tau=0$. The calculations are performed in the Holstein-Primakoff approximation, in which the spins and the resonator are described by harmonic oscillators, as explained in the Methods section. 
The system Hamiltonian is $H / \hbar=\omega_{B}(\Phi) a^{\dagger} a+\sum \omega_{\mathrm{j}} b_{\mathrm{j}}^{\dagger} b_{\mathrm{j}}+\sum i g_{\mathrm{j}}\left(b_{\mathrm{j}}^{\dagger} a-b_{\mathrm{j}} a^{\dagger}\right), g_{\mathrm{j}}$ being the coupling constant of spin $j$ with the resonator. We need to calculate $p(t)=|\alpha(t)|^{2}$ with $\alpha(t)=\left\langle 0\left|a(t) a^{\dagger}(0)\right| 0\right\rangle$, which represents the probability that a photon created at $t=0$ is still present at time $t$. As shown in [11] this quantity can be calculated by considering an effective non-Hermitian Hamiltonian

$$
H_{e f f} / \hbar=\left(\begin{array}{cccc}
\tilde{\omega}_{0} & i g_{1} & i g_{2} & \ldots \\
-i g_{1} & \tilde{\omega}_{1} & & \\
-i g_{2} & & \tilde{\omega}_{2} & \\
\vdots & & & \ddots
\end{array}\right) .
$$

with complex angular frequencies $\tilde{\omega}_{B}=\omega_{B}-i \kappa / 2$ and $\tilde{\omega}_{k}=\omega_{k}-i \gamma_{0} / 2 ;$ here, $\gamma_{0}$ is the spontaneous emission rate of each spin, and $\kappa=\omega_{B} / Q$ is the bus resonator damping rate (where we introduced its quality factor $Q$ ). Indeed, introducing the vector $X(t)$ of coordinates $\left[\left\langle a(t) a^{\dagger}(0)\right\rangle, \ldots,\left\langle b_{j}(t) a^{\dagger}(0)\right\rangle, \ldots\right]$ it can be shown that $d X / d t=-(i / \hbar) H_{\text {eff }} X$. The formal solution to this equation is then

$$
X(t)=\mathcal{L}^{-1}\left[\left(s+i H_{e f f} / \hbar\right)^{-1} X(0)\right],
$$

with $X(0)=x_{G}$ and $x_{G} \equiv(1,0, \ldots, 0)$. This implies that $\alpha(t)=x_{G}^{\dagger} \cdot X(t)=\mathcal{L}^{-1}\left[t_{1}(s)\right]$ with $t_{1}(s)=x_{G}^{\dagger} \cdot\left(s+i H_{e f f}\right)^{-1} \cdot x_{G}$ and $\mathcal{L}[f(s)]=\int e^{-s t} f(t) d t, s$ being a complex number. Since $t_{1}(s)$ is not singular on its imaginary axis, we only need $t_{1}$ for pure imaginary argument $s=-i \omega$ to perfom the Laplace transform inversion. As shown in [11], we have $t_{1}(-i \omega)=i /\left[\omega-\omega_{B}+i \kappa / 2-W(\omega)\right]$ with $W(\omega)=g^{2} \int \rho\left(\omega^{\prime}\right) d \omega^{\prime} /\left[\omega-\omega^{\prime}+i \gamma_{0} / 2\right]$. In this last formula, $g$ is the coupling constant of the spin ensemble to the bus resonator and $\rho(\omega)$ is the density of spins which is taken as explained below. Computing $\alpha(t)$ is thus achieved by evaluating $t_{1}$ for the distribution $\rho(\omega)$, and numerically evaluting the inverse Laplace transform. At the end of the calculation, we take the $\gamma_{0} \rightarrow 0$ limit since NV centers at low temperature have negligible energy relaxation.

\section{Single-photon Ramsey experiment (figure 4b)}

For the Ramsey-like experiment (figure $4 \mathrm{~b}$ ), each $\pi / 2$ pulse is realised by bringing the resonator and spins to resonance. For a fast pulse, the resonant interaction maps continu- 
ously the coherent state of the field to the superradiant mode in the spins. We calibrate the interaction time in such a way as to transform the state $x_{G}$ into the superposition $\frac{x_{G}-x_{S}}{\sqrt{2}}$. After the first $\pi / 2$ pulse, the resonator is kept detuned from the spin ensemble for a time $t$. The system state at this point can be evaluated using eq. (2). We define $X_{G}(t)$ (resp. $\left.X_{S}(t)\right)$ as the vector of coordinates $\left[\left\langle a(t) a^{\dagger}(0)\right\rangle, \ldots,\left\langle b_{j}(t) a^{\dagger}(0)\right\rangle, \ldots\right]$ at time $t$ with initial conditions $x_{G}$ (resp. $x_{S}$ ). A second $\pi / 2$ pulse is then applied before the amplitude $\alpha(t)$ of the field in the resonator is measured:

$\alpha(t)=\frac{1}{\sqrt{2}} x_{G}^{\dagger} \cdot U_{\pi / 2}\left(X_{G}(t)-X_{S}(t)\right)=\frac{1}{2}\left(x_{G}^{\dagger}+x_{S}^{\dagger}\right) \cdot\left(X_{G}(t)-X_{S}(t)\right)=\frac{1}{2} \mathcal{L}^{-1}\left(t_{1}(s)-t_{2}(s)+t_{3}(s)-t_{4}(s)\right)$

where $t_{2}(s)=x_{S}^{\dagger} \cdot\left(s+i H_{e f f}\right)^{-1} \cdot x_{S}$ and $t_{2}(s)=x_{S}^{\dagger} \cdot\left(s+i H_{e f f}\right)^{-1} \cdot x_{G}, t_{1}$ and $t_{4}$ are defined above. As shown in [11], $t_{2}(-i \omega)=-t_{1}(-i \omega) W(\omega)\left(s+i \tilde{\omega}_{0}\right) / g^{2}$ and $t_{3}=-t_{4}$.

\section{Parameters used in the simulation}

We thus see that the only thing that is needed to numerically perform these calculations is the density of spins $\rho(\omega)$ and the ensemble coupling constant $g$. The spin density $\rho(\omega)$ is chosen as the sum of three Lorentzian peaks separated by $2.3 \mathrm{MHz}$. The only parameters that are adjusted in order to fit the data are the coupling constants and the peak linewidth in the HF structure. As explained in the main text, we find that $g_{I} / 2 \pi=2.9 \mathrm{MHz}$ and $g_{I I I} / 2 \pi=3.8 \mathrm{MHz}$ fit best our data, with a linewidth of $1.6 \mathrm{MHz}$ for the spins belonging to group $I$, and $2.4 \mathrm{MHz}$ for the spins belonging to group $I I I$. We attribute the larger linewidth of the spins from group III to a residual misalignment of $B_{N V}$ with respect to the $[1,1,1]$ axis of the crystal which causes each of the three $<1,1,1>$ axes non-collinear with the field to undergo slightly different Zeeman shifts. A misalignment of $0.02 \mathrm{rad}$ would be enough to cause a broadening such as we observe.

We also note that the splitting of $2.3 \mathrm{MHz}$ between the three peaks of the HF structure is slightly larger than the value reported in most articles which is $2.18 \mathrm{MHz}$. Our data are however not precise enough to determine precisely whether this difference actually reflects 
a change in the HF interaction parameters of the NV center at low temperature.

[1] Gruber, A., et al., Scanning Confocal Optical Microscopy and Magnetic Resonance on Single Defect Centers, Science 276, 2012 (1997).

[2] Manson, N.B., et al., Nitrogen-vacancy center in diamond: Model of the electronic structure and associated dynamics, PRB 74, 104303 (2006).

[3] Dreau, A., et al., Avoiding power broadening in optically detected magnetic resonance of single NV defects for enhanced DC-magnetic field sensitivity, arxiv:quant-ph/1108.1178 (2011).

[4] Childdress, L., et al., Coherent Dynamics of Coupled Electron and Nuclear Spin Qubits in Diamond, Science 314, 281 (2006).

[5] Balasubramian, G., et al., Ultralong spin coherence time in isotopically engineered diamond, Nature Mater. 8, 383 (2009).

[6] Felton, S., et al., Hyperfine interaction in the ground state of the negatively charged nitrogen vacancy center in diamond, $P R B$ 79, 075203 (2009).

[7] van Wyk, J.A., et al., The dependences of ESR line widths and spin - spin relaxation times of single nitrogen defects on the concentration of nitrogen defects in diamond, J. Phys. D 30, 1790 (1997).

[8] Taylor, J.M., et al., High-sensitivity diamond magnetometer with nanoscale resolution, Nature Phys. 4, 810 (2008).

[9] Mallet, F., et al., Single-shot qubit readout in circuit quantum electrodynamics, Nature Phys. 5, 791 (2009).

[10] Lucero, E., et al., High-Fidelity Gates in a Single Josephson Qubit, Phys. Rev. Lett. 100, 247001 (2008).

[11] Diniz, I., et al., Strongly coupling a cavity to inhomogeneous ensembles of emitters : potential for long lived solid-state quantum memories, arxiv:quant-ph/1101.1842 (2011). 